\title{
Comparando o comportamento sexual de cegos e cegas diante das DSTs ${ }^{1}$
}

\section{Comparing the sexual behavior of blind men and women in view of STD}

\author{
Comparando el comportamiento sexual de ciegos y ciegas ante las EST
}

\author{
Kariane Gomes Cezario', Monaliza Ribeiro Mariano", Lorita Marlena Freitag Pagliuca'"I
}

\section{RESUMO}

Doenças sexualmente transmissíveis (DSTs) podem ser prevenidas por meio do comportamento e do sexo seguro. Objetivou-se compreender o comportamento de cegos e cegas diante da problemática das DSTs, comparando semelhanças e diferenças. Estudo descritivo realizado em uma associação de cegos de Fortaleza, entre setembro e novembro de 2005, com sete cegos e quatro cegas acima de 18 anos. A coleta de dados foi realizada por meio de entrevista utilizando-se questão norteadora e o tratamento destes deu-se via análise de conteúdo. Os dados foram organizados em dois temas: Uso do preservativo e Conhecimentos sobre saúde sexual. Quanto ao uso do preservativo para o sexo masculino, as alterações na sensibilidade, dificuldades práticas de uso e a confiança na parceira são causas de não uso, apesar da consciência sobre as DSTs. Entre as cegas, as causas são a confiança no parceiro justificada pelo não uso do preservativo, mas adotado pelo medo de gravidez indesejada. O tema Conhecimentos em saúde sexual confirma a superficialidade e presença de tabus e mitos para os dois sexos. Tais resultados alertam para a necessidade de iniciativas com enfoque na promoção da saúde de maneira acessível a esta população também exposta a fatores de risco para DSTs/AIDS.

Palavras chave: Portadores de deficiência visual; Doenças sexualmente transmissíveis; Comportamento sexual; Enfermagem.

\section{ABSTRACT \\ Sexually transmitted diseases (STD) can be prevented through safe behavior and sex. This study aimed to understand the behavior of blind men and women in view of the STD problem, comparing similarities and differences. A descriptive study was carried out in an association of blind people in Fortaleza, Brazil, between September and November of 2005, involving seven blind men and four blind women over 18 years old. Data were collected through}

interviews, using a guiding question and content analysis was used for data treatment. The data were organized in: Condom use and Sexual health knowledge. As to condom use for men, sensitivity changes, practical usage difficulties and trust in the partner are causes of non-use, despite awareness about STD. Among blind women, trust in the partner justified not using a condom. Women use condoms when they are afraid of an unwanted pregnancy. The Sexual health knowledge theme confirms the superficiality and presence of taboos and myths for both genders. These results alert about the need for initiatives focusing on health promotion in a way accessible to this population, which is also exposed to risk factors of STD/AIDS.

Key words: Visually impaired people; Sexually transmitted diseases; Sexual behavior; Nursing.

\section{RESUMEN}

Se pueden prevenir las enfermedades de transmisión sexual (ETS) mediante el comportamiento y el sexo seguro. La finalidad fue comprender el comportamiento de ciegos y ciegas ante la problemática de las EST, comparando similitudes y diferencias. Estudio descriptivo realizado en una asociación de ciegos de Fortaleza, Brasil, de septiembre a noviembre de 2005, con siete ciegos y cuatro ciegas con más de 18 años. La colecta de datos he sido realizada por entrevista con una cuestión orientadora y el análisis de contenido fue utilizado para tratar los datos. Los datos se organizaron en: Uso del preservativo y Conocimientos sobre salud sexual. En cuanto al Uso del preservativo para el sexo masculino, las alteraciones en la sensibilidad, dificultades

\footnotetext{
1 Trabalho realizado dentro do Projeto Saúde Ocular, UFC/CNPq.

Bolsista PIBIC/UFC. Estudante de Enfermagem. Fortaleza/Ceará. E-mail: kariane_gomes@yahoo.com.br.

II Bolsista PIBIC/UFC. Estudante de Enfermagem. Fortaleza/Ceará. E-mail: monalizamariano@yahoo.com.br. III Enfermeira, Doutora, Professora Titular da UFC, Pesquisadora CNPq. Fortaleza/Ceará. E-mail: pagliuca@ufc.br.
} 
prácticas de uso y la confianza en la pareja son causas de no uso, a pesar de la consciencia de las EST. Entre las ciegas, las causas son la confianza en la pareja justificada por el no uso del preservativo y adoptado por el miedo de embarazo indeseado. El tema Conocimientos en salud sexual confirma la superficialidad y presencia de tabúes y mitos para los dos sexos. Esos resultados alertan para la necesidad de

\section{NTRODUÇÃO}

O ser humano passa por várias fases no decorrer do seu desenvolvimento, entre elas a adolescência, quando ocorrem transformações psicológicas e corporais, além de alterações dos hormônios, resultando na exacerbação da libido, no amadurecimento e desenvolvimento dos órgãos sexuais. Conforme a literatura destaca, os adolescentes não apenas vivenciam essas mudanças, como passam a ser responsáveis por sua saúde e bem-estar ${ }^{(1)}$. Manifesta-se, então, o interesse pela sexualidade, acompanhado de dúvidas sobre gravidez, doenças sexualmente transmissíveis e sua prevenção.

Neste contexto, surge a discussão sobre a problemática de que o comportamento sexual envolve fatores de risco para as DSTS/AIDS. Como principais fatores mencionam-se: experiência e atividade sexual, idade, número de parceiros, freqüência das relações, estabilidade da atividade, modo de conseguir parceiros, desconhecimento acerca dos riscos, início precoce da vida sexual. Determinado estudo menciona, além destes fatores, o uso irregular do preservativo e o consumo de drogas lícitas e ilícitas ${ }^{(2)}$. Diante disso, os jovens necessitam de base educacional para a compreensão dos cuidados a serem tomados com vistas a preveni-los e se tornarem capazes de decidir sobre sua saúde, já que uma base educacional inadequada influi de forma decisiva para a percepção dessas entidades patológicas $^{(3)}$.

Atualmente, a preocupação dos profissionais da área da saúde com a sexualidade e suas conseqüências, como a transmissão de doenças e o risco de gravidez indesejada, entre outros, é evidente. Aos profissionais cabe, além do fazer técnico, desenvolver estratégias de promoção e educação em saúde adequadas à população, e iniciativas con enfoque en la promoción de la salud de manera accesible a esta población que también está expuesta a factores de riesgo para EST/SIDA.

Palabras clave: Personas con daño visual; Enfermedades de transmisión sexual; Conducta sexual; Enfermería.

aqui se volta para uma clientela específica: os cegos $^{(4)}$. Segundo se observa, a falta da visão não diminui o desenvolvimento e interesse sexual, apenas faz com que a curiosidade do cego sobre esse assunto torne-se diferenciada, pois cerca de $80 \%$ das interações com o mundo ao redor se dão através deste sentido. Neste caso, a perda visual é compensada pela exacerbação da sensação tátil e auditiva(4).

Contudo, pesquisas que relacionam os temas sexualidade e deficiência visual são escassas, provavelmente por se tratar de temática que envolve a superação de tabus, preconceitos, estigmas e exclusão, tanto por parte de profissionais de saúde quanto por educadores e responsáveis. Em relação à sexualidade e outros tipos de deficiência, prevalece literatura sobre as pessoas com algum grau de deficiência mental, seguido da deficiência auditiva ${ }^{(5-6)}$. A vivência da sexualidade dos filhos deficientes muitas vezes acaba sendo negada pelos pais, os quais negligenciam o diálogo e o fornecimento de informações ${ }^{(7)}$.

As conseqüências deste comportamento agravam-se quando aliadas à carência de materiais de educação em saúde adaptados e acessíveis aos cegos. Em estudo sobre a sexualidade de adolescentes com variados graus de deficiência visual observou-se grande déficit de conhecimentos sobre as formas de transmissão e contágio das DSTs, pois seus discursos apresentaram-se confusos, marcados por idéias vagas e difusas sobre a problemática $^{(8)}$. Estes fatos alertam para a necessidade de compreensão desta problemática entre os cegos bem como para a urgência em desenvolver métodos e materiais acessíveis a esta clientela. Em virtude de o processo de educação ultrapassar a mera transmissão de conhecimento e capacitar o 
indivíduo de modo a interferir em seu cotidiano e meio social, é pertinente a realização de estratégias para alcançar esta finalidade $\mathrm{e}^{(9)}$.

Cabe ao enfermeiro, portanto, como profissional de saúde que atua diretamente na promoção e educação em saúde da população, desenvolver estas estratégias com vistas ao empoderamento individual e coletivo e à melhora na qualidade de vida da clientela. Contudo, estas ações devem ser precedidas pela compreensão da realidade e das demandas específicas de cada clientela. Deste modo, este estudo teve como objetivo compreender o comportamento de cegos e cegas diante da problemática das DSTs, comparando suas semelhanças e diferenças.

\section{METODOLOGI A}

Trata-se de estudo do tipo exploratóriodescritivo, com abordagem qualitativa. Desenvolveu-se entre setembro e novembro de 2005 em uma associação de cegos no município de Fortaleza-CE. Esta associação, entidade sem fins lucrativos, destina-se à promoção da pessoa cega por meio de atividades de ensino regular e educação de jovens e adultos, cursos profissionalizantes, além de atividades culturais e sociais em geral.

Os sujeitos da pesquisa foram mulheres e homens cegos, com idade a partir de 18 anos. Os critérios de inclusão dos sujeitos no estudo foram: ter idade a partir de 18 anos, para evitar a necessidade de autorização de pais ou responsáveis, e disponibilidade para participar da pesquisa. Quanto à limitação do número de participantes, deu-se pelo método de saturação ${ }^{(10)}$.

A coleta de dados ocorreu mediante entrevista aberta com a questão norteadora: Comente a sua vida sexual e os cuidados que você toma para não adquirir doença sexualmente transmissível. Ao expressarem interesse em participar do estudo, após convite aberto entre membros da instituição, as entrevistas eram agendadas e realizadas em sala disponibilizada na associação, a fim de garantir a privacidade. Estas entrevistas eram gravadas com o consentimento dos sujeitos, e posteriormente transcritas e organizadas. A análise dos dados, após escuta repetida e transcrição literal, fez-se pelo método de análise de conteúdo, o qual utiliza procedimentos sistemáticos e objetivos de descrição do conteúdo das mensagens, permitindo a construção de categorias temáticas ${ }^{(10)}$. Desse modo os resultados organizaram-se em temas e categorias.

Solicitou-se o consentimento da referida associação e, logo após, o projeto foi aprovado pelo Comitê de Ética e Pesquisa do Complexo Hospitalar da Universidade Federal do Ceará conforme protocolo no 196/05. Providenciou-se o termo de consentimento livre e esclarecido dos sujeitos, o qual foi lido e explicado pelas pesquisadoras e, em seguida, assinado pelo participante, contando com a presença de uma testemunha vidente que também o assinou.

\section{RESULTADOS}

Participaram da pesquisa onze cegos, dos quais quatro mulheres e sete homens na faixa etária entre 19 e 41 anos. Em relação à escolaridade, nove completaram o ensino médio. Sobre o estado civil, dois entrevistados eram casados, os demais solteiros ou solteiros com relacionamento estável. No Quadro 1, são apresentados os resultados da análise das falas, divididos nos dois temas, Uso do preservativo e Conhecimento sobre saúde sexual, com suas respectivas categorias. 
Quadro 1: Análise comparativa do comportamento sexual

entre cegos e cegas diante das DSTs. Fortaleza (CE), 2005.

\begin{tabular}{|c|c|}
\hline CEGOS & CEGAS \\
\hline TEMA 1: Uso do preservativo & TEMA 1: Uso do preservativo \\
\hline Por que não usa & Por que não usa \\
\hline - Relacionamentos Estáveis/Confiança & - Relacionamentos Estáveis/Confiança \\
\hline $\begin{array}{l}\text {... nós dois nos confiamos .... a gente não utiliza. } \\
\ldots \text { gosto de parceiras fixas exatamente pra não ser } \\
\text { preciso usar. }\end{array}$ & $\begin{array}{l}\text { Mas quando você tem um parceiro fixo acha que não há } \\
\text { tanta necessidade. } \\
\text { Eu tenho um namorado fixo, só tenho relação com ele, e a } \\
\text { gente não usa camisinha. }\end{array}$ \\
\hline - $\quad$ Alterações na Sensibilidade & - $\quad$ Alterações na Sensibilidade do Parceiro \\
\hline $\begin{array}{l}\text {... tira um pouco da sensibilidade... não acho legal } \\
\ldots \text {.. impossibilita até de haver gozo sexual da minha } \\
\text { parte. }\end{array}$ & $\begin{array}{l}\text { Porque o meu parceiro não gosta; ele não gostava, ele } \\
\text { não se dá. } \\
\text { Porque eu sei que a maioria não gosta de usar } \\
\text { preservativo. }\end{array}$ \\
\hline \multicolumn{2}{|l|}{ Por que usa } \\
\hline - Prevenção de DST/AIDS & - $\quad$ Prevenção de Gravidez Indesejada \\
\hline $\begin{array}{l}\text {... acho que não tem nada melhor do que você se } \\
\text { prevenir (...) para evitar que a gente pegue esse } \\
\text { tipo de doença. } \\
\text {... se não usar, com certeza, a gente irá sofrer danos } \\
\text { maiores... }\end{array}$ & $\begin{array}{l}\text { Eu perdi a virgindade com ele, mas usando preservativo. } \\
\text { Eu acho que não era tanto com medo de doença, mas } \\
\text { com medo de gravidez (risos), mais de gravidez do que } \\
\text { de doença. }\end{array}$ \\
\hline TEMA 2: Conhecimento em saúde sexual & TEMA 2: Conhecimento em saúde se \\
\hline Família & Família \\
\hline - $\quad$ Falta de Diálogo & - Falta de Diálogo \\
\hline $\begin{array}{l}\text {... na minha casa eu não tive contato com esse tipo } \\
\text { de conversa. }\end{array}$ & $\begin{array}{l}\text { Na família não. } \\
\text { Já conversam, toma cuidado para não engravidar. } \\
\ldots \text { alertar sobre doença não. A preocupação maior deles } \\
\text { é engravidar. }\end{array}$ \\
\hline - $\quad$ Choque de Valores & - $\quad$ Choque de Valores \\
\hline $\begin{array}{l}\text {...eles são totalmente contra (...) eles encaram } \\
\text { como imoralidade... }\end{array}$ & $\begin{array}{l}\text { Não com tanta liberdade, porque meus pais já são bem } \\
\text { antigos. }\end{array}$ \\
\hline \multicolumn{2}{|l|}{ Sociedade } \\
\hline \multirow{2}{*}{\multicolumn{2}{|c|}{$\begin{array}{l}\text { Campanhas educativas, na imprensa (...) pegava } \\
\text { algumas coisas, mas com dúvidas. }\end{array}$}} \\
\hline & \\
\hline - Escola & - Escola \\
\hline $\begin{array}{l}\text {... eu não tive coragem de perguntar e de } \\
\text { questionar, aí fiquei sem entender mesmo (acerca } \\
\text { de uma palestra na escola). }\end{array}$ & $\begin{array}{l}\text { Leio, ou então documentário. } \\
\text {...assisti palestras, no ginecologista também, na } \\
\text { escola, em feiras de ciência, em congressos. }\end{array}$ \\
\hline
\end{tabular}

\section{SCUSSÃO}

\section{TEMA 1: Uso do preservativo}

Apesar da relevância no combate à AIDS e demais DSTs, o uso do preservativo fica comprometido, entre os homens, pela confiança na parceira sexual, nas queixas de alterações na sensibilidade e no prazer e pelo fato de considerarem o seu uso pouco prático ou não gostarem. Já entre as mulheres, conforme mostram os depoimentos, o uso do preservativo não é freqüente, principalmente pela confiança no parceiro e porque este rejeita o seu uso, confirmando os fatores já revelados pelos cegos. Torna-se, então, um fator de risco considerável para a transmissão das DSTs, o qual merece correta abordagem da educação em saúde.

O senso comum tem induzido as pessoas a considerarem que relacionamentos estáveis e confiança no parceiro sexual são suficientes como formas de proteção às doenças sexualmente transmissíveis. Mesmo entre aqueles que estavam sem parceira fixa, a preferência era pelas relações sexuais sem uso de preservativo. Como menciona a literatura, no 
caso dos relacionamentos estáveis, nos quais estão em discussão aspectos como amor, paixão, cumplicidade, há uma sensação de

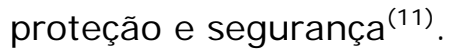

Como observado, o relato das cegas sobre a confiança no parceiro foi unânime, e exaltado várias vezes. A confiança está inserida em um contexto no qual se almeja um relacionamento completo, sem lacunas. Este desejo foi característico entre as mulheres, pois elas valorizam o amor e deixam-se envolver pela emoção. Enquanto as mulheres dão importância aos relacionamentos amorosos, os homens vivenciam o relacionamento como atributo individual, em favor da experiência da sexualidade ${ }^{(12)}$.

A confiança no parceiro sexual induz ao não uso do preservativo e expõe a mulher à contaminação, fato confirmado pelo número crescente de mulheres casadas que contraíram HIV. Como mostra a realidade, a epidemia do HIV/AIDS está se heterossexualizando(13). Porém essa exposição não se restringe à AIDS, pois se estende às DSTs em geral. Há, pois, um aumento acentuado de DSTs no sexo feminino ${ }^{(14)}$.

De modo geral, os cegos e as cegas sentem-se influenciados negativamente pelas alterações da sensibilidade ocasionadas pelo uso do preservativo, como algo prejudicial ao prazer e ao desempenho sexual. Preferem que o contato pele a pele seja direto, como forma de obter o máximo de prazer sexual. Conforme a literatura, em estudos com pessoas videntes, também há relato de recusa do uso do preservativo por impedir o prazer pleno, e tornar a relação frustrante. Seu uso prejudica sexualmente a harmonia do encontro do casal $^{(15)}$.

Entre as cegas, há resistência ao uso do preservativo em virtude da falta de diálogo ou de acordo entre o casal. A negociação do uso do preservativo entre os parceiros tem sido uma das maiores dificuldades da população feminina quanto à utilização de um método de barreira nas relações sexuais ${ }^{(16)}$. Embora o diálogo seja um veículo capaz de proporcionar a melhoria do relacionamento, redunda, muitas vezes, em obstáculo. Entretanto, a falta de diálogo constitui agravante nas dificuldades em relação à sexualidade ${ }^{(17)}$.
Em determinada fala, a cega refere já saber que nenhum homem gosta de usar preservativo. Ela pressupõe a negação e não sugere seu uso durante a relação sexual pelo receio de uma negação. Segundo defende, os homens não possuem a característica de seguir normas tradicionais e a literatura afirma que as mulheres aderem mais às normas tradicionais do que os homens ${ }^{(11)}$. No decorrer do discurso, algumas mulheres relatam 0 uso do preservativo esporadicamente.

$\mathrm{Na}$ opinião dos homens, a colocação do preservativo quando o casal está envolvido nas carícias preliminares é motivo de não uso, pois temem a perda da ereção, associada à falta de habilidade de colocar o preservativo, quebrando o clima do momento. Acrescidas a estes fatores, há afirmações categóricas de não gostarem do preservativo, e até evitar as relações sexuais a ter de adotá-lo. Um dos entrevistados conhece o preservativo feminino, mas rejeita seu uso. Isto reforça a pertinência de ações efetivas que abordem sua importância no combate às DSTs, tornando-o de maior conhecimento da população e adesão ao uso(16).

Apesar dos pontos desfavoráveis há relatos de valorização do preservativo como uma prática segura, instrumento de prevenção, necessário à manutenção de uma vida sexual saudável. Acreditam no valor da prevenção e vêem no preservativo um meio para ser esse objetivo alcançado, embora a maioria não use ou não goste. Além disso, a literatura menciona a AIDS como a doença que mais assusta pela impossibilidade de cura e iminência de morte. É defendido que quem estima sua saúde deve usá-lo sempre ${ }^{(15)}$. Em outro estudo, a preocupação de uma gravidez indesejada é o estímulo ao uso do preservativo, mas nenhum dos seus entrevistados menciona a intenção de se prevenir de DST/AIDS ${ }^{(13)}$.

Entre as cegas a justificativa para adotar o uso do preservativo foi a prevenção de uma gravidez indesejada. Esta argumentação é freqüente entre os jovens. Desse modo, mais uma vez ficam expostos às DSTs/AIDS ${ }^{(14)}$. Contudo, conforme parece, mesmo quando há o uso do preservativo, este não se dá de forma adequada. Diante disso, a consciência do perigo e a possibilidade de contrair uma DST estão 
presentes. De acordo com os discursos das cegas, estes são motivos para o seu uso.

É freqüente a associação entre a utilização do preservativo e a prevenção de DST. Por conseguinte, conforme se acredita, a maioria das pessoas usa e sabe a importância da prevenção. Entretanto, há o pensamento errôneo de que o problema é do outro e que a situação jamais vai acontecer consigo. Como afirma a literatura, essa idéia funciona como anteparo para se sentirem seguros. $O$ fato de ser entendido como "doença dos outros" serve como justificativa para não se sentirem vulneráveis $^{(13)}$. Apesar do não uso do preservativo ser claro nas falas, há consciência da importância da prevenção, pois eles sabem o que devem fazer para evitar contrair uma DST. Todavia admitem não estarem tomando o devido cuidado.

No comportamento dos entrevistados existe contradição, evidenciada quando sabem e reconhecem a importância da prevenção como um meio de autocuidado em suas vidas, porém rejeitam de maneira unânime o preservativo, e se permitem correr o risco de adquirir doença passível de trazer graves complicações. Concorda-se, portanto, que deveria prevalecer a ênfase da prevenção, pelo uso do preservativo, como uma forma consciente de exercer a sexualidade de maneira prazerosa e com segurança, evitando o contágio de DSTs e $\operatorname{AIDS}^{(16)}$.

\section{TEMA 2: Conhecimento em saúde sexual}

Conforme se pode notar o desconhecimento sobre a temática DST é geral; as pessoas possuem apenas informações superficiais. Entretanto, para se gerar uma cultura de prevenção e combate às DSTs/AIDS, é relevante se divulgar conhecimento acerca desta realidade: tipos, fatores de risco, sinais e sintomas, maneiras de prevenção.

Segundo se afirma, entre a população menos favorecida de educação e informações há maior vulnerabilidade para acometimentos na saúde em geral, inclusive a sexual ${ }^{(18)}$. Ora, o cego poderia se enquadrar nessa população, pois está de certo modo em desvantagem em relação aos meios de comunicação e materiais educativos para DST/AIDS, além de fatores como preconceitos e estigma. Deste modo, deve demandar mais cuidado em relação à saúde sexual por parte dos serviços de saúde para que a difusão de conhecimento chegue a todos.

A família é a principal responsável pela educação e formação dos filhos. Constitui o alicerce, juntamente com a escola, para o desenvolvimento e aprendizado. Conforme se sabe, o diálogo na família representa uma forma de troca de experiência e aprendizado. Como exposto pelas cegas, não existe diálogo na família, assim, as curiosidades e dúvidas são supridas, muitas vezes, com amigos. Entre os familiares, a conversa limita-se aos irmãos. Em uma das falas uma cega relata conversar com o irmão e juntos assistirem a filmes eróticos por curiosidade.

No contexto da família ficou evidente a falta de diálogo entre os entrevistados e os seus pais, principalmente. Mas o estímulo ao debate sobre esta temática no ambiente familiar contribui diretamente para ser a sexualidade exercida pelos filhos de maneira saudável e segura, e isto influirá diretamente sobre a questão das DSTS/AIDS, e poderá diminuir os comportamentos de risco, porquanto se aprenderia desde cedo sobre os prejuízos causados por estas doenças. Dessa forma, seria possível gerar um comportamento favorável ao sexo seguro ${ }^{(19)}$.

Tal falha na comunicação contribui para que os filhos procurem informações em outros meios, correndo o risco de aprenderem de maneira inadequada, pois ninguém como os pais para educarem os seus filhos na construção de valores sexuais ${ }^{(17)}$. Em determinado depoimento, conforme o cego afirma, a dificuldade está na pessoa em si. Isto leva a pensar que um possível constrangimento em se abordar a temática não reside somente na família, mas também no próprio cego.

Para os entrevistados, os choques de valores apresentam-se como fator relevante e gerador de certo incômodo. As diferenças de valores, choque de gerações, tornam-se barreiras ao diálogo. Essas diferenças podem ser decorrentes da dificuldade dos pais em se adaptarem às modificações acontecidas pelas mudanças culturais entre seu processo de criação e o atual contexto dos seus filhos devido à mudança nos valores sexuais e religiosos. 
Conversar sobre sexualidade e DST/AIDS é visto pelos pais, segundo os relatos, como uma atitude vergonhosa, uma imoralidade.

De modo geral, a sociedade é apontada como principal meio informativo e gerador de opiniões entre os entrevistados. É daí que vem a informação mesmo quando transmitida de modo pouco esclarecedor ou superficialmente. Os meios de comunicação têm papel significativo neste processo de apreensão de conteúdo, pois são os chamados "veículos de massa", como televisão, rádio, jornais, revistas, que atingem ao mesmo tempo uma grande parte da população, mediante veiculação das campanhas educativas sobre DST/AIDS, procurando influenciar o comportamento e a maneira de agir em relação à vida. Como evidenciado, o cego demonstrou não se excluir desse processo ${ }^{(15)}$.

Apesar da limitação sensorial do cego, a televisão foi citada como meio de aprendizado e, diante dessa atitude, constatou-se a capacidade de adaptação do cego: mesmo impossibilitado de ver imagens sobre a temática, é capaz de absorver e apreender os conteúdos das mensagens. Isto confirma que o processo de comunicação envolve uma percepção seletiva de interpretação de conteúdo. As pessoas percebem, absorvem e lembram o conteúdo de diversas maneiras ${ }^{(15)}$.

O conhecimento veiculado na imprensa é apreendido por meio de campanhas, as quais, apesar do seu caráter informativo, utilizam um tom muito mais de acusação e repressão do que de estímulo a atitudes de prevenção e autocuidado, advindo daí idéias equivocadas sobre DST/AIDS. Ressaltar a morbidade e mortalidade da AIDS, em detrimento da prevenção, levou ao surgimento e expansão da pandemia e à segregação social das pessoas infectadas $^{(18)}$.

As informações sobre DSTs geralmente são adquiridas fora do ciclo familiar. Como adverte a literatura, o sexo e a sexualidade são aprendidos com amigos, no pátio da escola, ou na vida familiar, e assim comprova-se a freqüente inadequação dessa educação informal ${ }^{(14)}$.

Alguns obtêm informação na escola. Esta, porém, quase sempre é superficial e não enfoca a prevenção. Outras vezes os jovens são esclarecidos por profissionais de saúde, como o ginecologista. Defende-se $\mathrm{o}$ direito à informação, com acesso aos meios para obtê-la, atingindo o mais elevado padrão da saúde sexual e reprodutiva ${ }^{(16)}$.

Nos depoimentos, a participação de conhecidos e amigos foi valorizada por meio de conversas com quem possuem mais afinidade e confiança, quando se sentem à vontade para trocar experiências e esclarecer dúvidas. Com os amigos e com as experiências sexuais que vivenciam ao longo da vida, vão "se virando como podem". Para os participantes deste estudo, também são importantes pesquisas como estas, pois constituem difusor de conhecimentos sobre a temática e apreensão de conteúdos. Nesse estudo, conforme observado, apesar de já terem algum conhecimento sobre a temática, este é essencialmente teórico, decorrente de explanação verbal, o que limita sua apreensão.

\section{COMENTÁRI OS FI NAIS}

Segundo relataram os cegos e as cegas, o uso do preservativo não é uma prática freqüente em suas relações. Entre os homens, a confiança nas parceiras sexuais, as alterações de sensibilidade e a pouca praticidade motivaram seu não uso. Entre as mulheres, a confiança no parceiro e o fato de este não gostar de usar o preservativo são fatores decisivos para este posicionamento. Tal comportamento de risco apóia-se no baixo nível de conhecimento em educação sexual, influenciado por dificuldades com a família e no meio social onde estão inseridos.

Contudo, consideram a atitude da prevenção relevante no controle às DSTs/AIDS, mostrando divergência entre o discurso e o comportamento sexual adotado. Esta conclusão não se distancia dos resultados encontrados por outros estudos entre pessoas videntes. Portanto, o comportamento sexual dos cegos assemelha-se ao do restante da população. Mas ainda que os problemas tenham aspectos comuns aos videntes, os cegos merecem abordagens próprias para pleno usufruto dos conteúdos para promoção da saúde.

Tais resultados alertam para a necessidade de iniciativas com enfoque acessível a esta população que também está 
exposta às DSTs/AIDS. Desse modo, para maior efetividade das ações de educação em saúde dirigidas aos cegos, são pertinentes abordagens interativas nas quais o diálogo seja explorado como instrumento de aprendizagem. A apresentação de conteúdos para serem discutidos individualmente ou em grupos de cegos pode ser uma estratégia a ser explorada. Associados ao diálogo, materiais tácteis também instigam o interesse, haja vista que a apreensão de objetos e o próprio sistema de escrita Braille desenvolvem este sentido.

\section{REFERÊNCI AS}

1. Marques ES, Mendes DA, Tornis NHM, Lopes CLR, Barbosa MA. O conhecimento dos escolares adolescentes sobre doenças sexualmente transmissíveis/AIDS. Revista Eletrônica de Enfermagem [Internet]. 2006 [cited $2007 \mathrm{fev}$ 05];8(1):58-62. Available from: http://www.fen.ufg. br/revista/revista8 1/origin al_07.htm.

2. Silva PDB, Oliveira MDS, Matos MA, Tavares VR, Medeiros $M$, Brunini $S$, et al. Comportamento de risco para doenças sexualmente transmissíveis em adolescentes escolares de baixa renda. Revista Eletrônica de Enfermagem [Internet]. 2005 [cited $2007 \mathrm{fev}$ 05]; 7(2):185-9. Available from: http://www.fen.ufg.br/revista/revista7_2/origin al_06. htm.

3. Passos MRL. Doenças sexualmente transmissíveis. 4a edição. Rio de Janeiro: Cultura Médica; 1995.

4. Costa EM, Castro DN, Pagliuca LMF. A assistência de enfermagem: percepção da pessoa cega-reflexão sobre ética e solidariedade. Revista Brasileira de Enfermagem. 1999; 52(4):615-23.

5. Maia ACB, Camossa DA. Relatos de jovens deficientes mentais sobre a sexualidade através de diferentes estratégias. Paidéia. 2002; 12(24): 205-14.

6. Barroso MGT, Aguiar MIF, Oliveira KF. Educação na prevenção das DST com familiares de portador de deficiência auditiva. DST - Jornal Brasileiro de Doenças Sexualmente Transmissíveis. 2001;13(2): 18-22.

7. Salzedas PL, Bruns, MAT. Adolescer: a vivência de portadores de deficiência visual. Revista Benjamim Constant. 1999;5(12):6-16.
8. Moura GR, Pedro ENR. Adolescentes portadores de deficiência visual: percepções sobre sexualidade. Revista Latino-americana de Enfermagem. 2006; 14(2):220-26.

9. Santos AS. Health education: reflection and applicability in primary heath care. Online Brazilian Journal of Nursing [Internet] 2006 [cited 2006 out 20];5(2). Available from: http://www.uff. br/objnursing/index.php/nursing /article/view/435/102.

10. Bardin L. Análise de Conteúdo. Lisboa: Edições 70; 2004.

11. Amorim MM, Andrade AN. Relações afetivosexuais e prevenção contra infecções sexualmente transmissíveis e aids entre mulheres do município de Vitória-ES. Psicologia em Estudo. 2006; 11(2):331-9.

12. Torres CA, Beserra EP, Barroso MGT. Relações de gênero e vulnerabilidade às doenças sexualmente transmissíveis: percepções sobre a sexualidade dos adolescentes. Escola Anna Nery Revista de Enfermagem. 2007; 11(2): 296-302.

13. Guerriero I, Ayers JRCM, Hearst N. Masculinidade e vulnerabilidade ao HIV de homens heterossexuais em São Paulo, SP. Revista de Saúde Pública. 2002;36(4 Suppl): 50-60.

14. Okawara H. Educação sexual. In: Halbe HW, editor. Tratado de ginecologia. 3a edição. São Paulo: Roca; 2000. p. 112-126

15. Camargo BV, Barbará A. Efeitos de panfletos informativo sobre AIDS em adolescentes. Psicologia: Teoria e Pesquisa. 2004; 20(3):279-87.

16. Santos CL, Pessoa IN, Pereira PFQ, Ferreira TF. Preservativo feminino: uma nova perspectiva de proteção. Revista de Enfermagem da UERJ. 2005; 13:270-4.

17. Borges ALV, Nichiata LYI, Schor N. Talking about sex: the social and familial net as a base for sexual and reproducive health promotion among adolescents. Revista Latino-Americana de Enfermagem [Internet]. 2006 [cited 2008 jul 05]; 14(3):422-7. Available from: http://www.scielo.br/pdf/rlae/v14n3/v14n3a17.pdf.

18. Gonçalves EM, Varandas R. O papel da mídia na prevenção do HIV/AIDS e a representação da mulher no contexto da epidemia. Ciência e Saúde Coletiva. 2005; 10(1):229-35. 
19. Bastos OM, Deslandes SF. Sexualidade e o adolescente com deficiência mental: uma revisão bibliográfica. Ciência e Saúde Coletiva. 2005; 10(2): 389-97.

Artigo recebido em 03.07.07

Aprovado para publicação em 30.09.08 